CENTRO UNIVERSITÁRIO DE BRASÍLIA

FACULDADE DE CIÊNCIAS DA SAÚDE

\title{
A INTELIGÊNCIA ARTIFICIAL A CAMINHO DA VISÃO DA EXPERIÊNCIA DO PACIENTE
}

Julia Correia da Costa Barros

Brasília 


\title{
A INTELIGÊNCIA ARTIFICIAL A CAMINHO DA VISÃO DA EXPERIÊNCIA DO PACIENTE
}

\author{
Julia Correia da Costa Barros
}

\section{RESUMO}

O estudo tem como escopo central examinar o impacto da inteligência artificial na experiência do paciente, a qual pode ser entendida como a soma das interações que ocorrem entre o paciente e os serviços de saúde. A substituição de ferramentas e métodos de gestão ultrapassados por sistemas e tecnologias inovadoras, como a inteligência artificial, aumentam a qualidade dos serviços prestados em saúde e ampliam a segurança dos pacientes. Busca-se uma revisão integrativa com o objetivo de analisar o impacto da inteligência artificial na experiência do paciente. O referencial teórico do estudo ora apresentado toma como base duas linhas institucionais contemporâneas. Trata-se de um trabalho de natureza qualitativa (descritiva e interpretativa), desenvolvido com base em dados documentais. Para a triagem inicial de artigos da área da saúde, utilizou-se as bases de dados LILACS e Medline. Das amostras levantadas, foram selecionados cinco artigos, os quais permitem apontar a relevância do uso de novas tecnologias na experiência do paciente, embora se possa reconhecer que o número de pesquisas que abordam a temática ainda seja incipiente, talvez pela natureza somente descritiva. Não obstante, considera-se que o estudo aqui levado a cabo possa significar uma contribuição para que novas pesquisas sejam realizadas, não somente com dados documentais, mas, sobretudo, com dados empíricos, que permitam dar voz ao paciente para se chegar a uma visão mais naturalística da questão.

Palavras-chave: experiência do paciente; cuidados em saúde; inteligência artificial 


\section{Introdução}

O presente estudo tem como escopo central examinar o impacto da inteligência artificial na experiência do paciente. Nos últimos anos, a percepção de qualidade e excelência no cuidado prestado por organizações de saúde tem ido além da parte clínica, uma vez que a experiência do paciente passa a constituir um indicador relevante $^{1}$. O referencial teórico do estudo ora apresentado toma como base duas linhas institucionais contemporâneas, as quais são elencadas a seguir, ainda que de maneira sucinta.

Em primeiro lugar, para o reconhecido The Beryl Institute, a experiência do paciente pode ser entendida como a soma de todas as interações que ocorrem entre o paciente e os serviços de saúde e como o paciente avalia essas interações. ${ }^{2}$ Essa caracterização surgiu a partir de revisão de pesquisas e questionamentos, junto a acompanhantes e pacientes para se identificar os principais elementos que poderiam nortear o trabalho clínico. Hoje, os principais temas giram em torno de percepções dos pacientes e familiares em toda continuidade do cuidado, interações pessoais e cultura organizacional das instituições. A definição foi elaborada, validada e aperfeiçoada pela comunidade de profissionais do Instituto acima referido ${ }^{1}$.

Em segundo lugar, o Institute for Health Care Improvement (IHI) destaca que todos, em uma dada instituição, desde a recepção ao diretor geral, desempenham um papel na construção da jornada de um paciente. Ao considerar a experiência de atendimento um momento de respeito, cooperação, tomada de decisão compartilhada, transições bem coordenadas e eficiência, os hospitais concebem melhorias nos resultados de avaliação da prestação de cuidados ${ }^{3}$.

A experiência dos pacientes é única e impactada pelas várias particularidades que compõem os encontros de assistência médica, como ambiente, interações clínicas e características da gestão do cuidado. Dessa forma, a "soma de todas as interações" da definição retrata a natureza interativa e dinâmica da experiência do paciente. ${ }^{1}$ Nesse sentido, o IHI apresentou, em 2008, o conceito de triple aim (tripla meta), uma estrutura desenvolvida para descrever novas abordagens que otimizem o desempenho dos sistemas de saúde. O modelo baseia-se na ideia de melhorar a saúde da população, valorizar a experiência do paciente, enquanto reduz ou estabiliza o custo per capita com saúde ${ }^{4}$. 
O triple aim é um esforço integrado entre qualidade, segurança, custos e resultados assistenciais com os pacientes no centro do cuidado. Seus princípios incluem planejar qualidade na assistência ao paciente, enquanto reduz custos desnecessários de exames, internações e cirurgias ${ }^{4,5}$. Parece que a conquista bem sucedida do modelo requer organizações de saúde altamente eficazes, sendo fundamental o engajamento de todos nos processos estabelecidos para um trabalho satisfatório e produtivo ${ }^{6}$.

A participação da experiência do paciente como um indicador de qualidade tem sido um interesse que, gradativamente, ganha importância nas instituições de saúde. Assim, ouvir e aprender com a experiência do paciente possibilita ao médico uma melhor prestação de cuidados, além de produzir dados que beneficiam as organizações de saúde com foco no cliente ${ }^{7}$.

Nessa perspectiva, a automação de processos, substituição de ferramentas e métodos de gestão ultrapassados por sistemas e tecnologias inovadoras, que aumentem a qualidade dos serviços prestados, possibilita o desenvolvimento de soluções diversificadas, que ampliam a segurança dos pacientes, reduzem erros e reconhecem falhas nos setores ${ }^{8}$. A transformação digital leva ao desenvolvimento contínuo de uma cultura inovadora atrelada a uma melhor experiência do paciente como principais pilares da assistência à saúde. Assim, as tecnologias em saúde devem ser avaliadas considerando o papel que elas podem desempenhar para melhorar o atendimento, impulsionando a eficácia do cuidado ${ }^{8,9}$.

Ressalte-se que, ao longo dos anos, a capacidade de processar e armazenar dados aumentou exponencialmente com o uso da Inteligência Artificial (IA), ferramenta que oferece uma nova e potencialmente benéfica solução para a prevenção e o gerenciamento de doenças ${ }^{10}$. A IA é uma área de pesquisa da ciência da computação que busca desenvolver sistemas para reproduzir a capacidade humana de resolução de problemas e tomada de decisões a partir de algoritmos definidos por especialistas na matéria ${ }^{11}$.

A integração da IA e tecnologias relacionadas à área médica traz inúmeros benefícios, com maior organização e segurança no armazenamento de informações, auxílio no diagnóstico, com interpretação de laudos e exames de imagem, tecnologias de telecomunicação para fornecer à distância informações para médicos e pacientes e associação de sintomas por sistemas para apoio à decisão clínica ${ }^{12}$. 
Nessa perspectiva, para impactar positivamente a experiência do paciente, precisamos pensar abordagens que permitam correlacionar as interações do indivíduo com o sistema de saúde ao atual contexto de transformação digital. Com base no tripé teórico sumarizado acima, a presente investigação toma como propósito levantar e discutir evidências disponíveis na literatura da área da medicina sobre o tema, estabelecer bases para estudos futuros e somar esforços para a melhoria da assistência de saúde. Para tanto, caminhos teóricos metodológicos foram selecionados, o que será apresentado a seguir.

\section{Metodologia}

Uma metodologia requer um direcionamento teórico que, via de regra, pode-se enveredar por dois caminhos: um de natureza qualitativa e outro de natureza quantitativa. Em ciências sociais, como em todo sistema, um corpus, é um princípio que cresce, daí a necessidade que todo cientista da área de humanas deve estar ciente da primeira lição primordial de uma seleção qualitativa: proceder por etapas, ou seja, selecionar; analisar; selecionar de novo ${ }^{13}$ (Bauer \& Arts, 2000).

O estudo ora desenvolvido pode ser considerado de natureza qualitativa (descritiva e interpretativa), uma vez que envolve uma revisão integrativa da literatura, com base em amostras documentais coletadas no PubMed e na Biblioteca Virtual em Saúde (BVS). Buscou-se elaborar a revisão com base em caminhos balizados por cinco etapas:

- elaboração da questão norteadora;

- estabelecimento de critérios de inclusão e exclusão de artigos (seleção da amostra);

- definição de dados a serem extraídos dos artigos selecionados; avaliação dos textos incluídos;

- $\quad$ análise dos resultados;

- discussão e apresentação da revisão.

Para guiar o estudo, formulou-se, ainda, a seguinte questão: como a inteligência artificial aplicada à saúde pode transformar a experiência do paciente? 
Na seleção de artigos científicos da área médica, foram empregadas duas bases de dados, com intuito de ampliar o âmbito da pesquisa, a saber: Medical Literature Analysis and Retrievel System Online (MEDLINE) e Literatura Latino-americana e do Caribe em Ciências da Saúde (LILACS). Mediante a utilização dessas ferramentas, pude selecionar fontes relativamente recentes voltadas para o tema ora pesquisado.

Ressalte-se que a BVS envolve um acervo composto por fontes de informação em ciências da saúde para atender às necessidades de informação científico-técnica de profissionais e estudantes da área sob a coordenação do Centro Latino-Americano de Informações em Ciências da Saúde (BIREME) ${ }^{14}$. Já a MEDLINE é uma base de dados da literatura internacional da área médica e biomédica, produzida pela National Library of Medicine (NLM), que contém referências bibliográficas e resumos de títulos de revistas publicadas nos Estados Unidos e em outros setenta países. A base de dados MEDLINE é única, podendo variar a interface de pesquisa adotada. O PubMED é a interface adotada pela NLM ${ }^{14}$. Por outro lado, a LILACS é uma base de dados cooperativa do Sistema BIREME, que compreende a literatura relativa às ciências da saúde, publicada nos países da região América Latina e Caribe e pode apresentar dados de estudos condizentes com a realidade brasileira ${ }^{14}$. Paralelo às fontes selecionadas, buscou-se outras referências complementares, como forma de subsídio de caminhos metodológicos, tais como a "saturação" de dados. ${ }^{1}$

Outro aspecto que merece ser levado em conta é a noção de contexto. Nas palavras de Silva ${ }^{15}$ (2019):

Na atualidade, correntes dos estudos críticos do discurso comungam a noção de contexto que envolve, de maneira simultânea, uma dimensão cognitiva e uma dimensão social/.../. Contextos são construtos mentais que afloram de acordo com conhecimentos adquiridos socialmente, bem como com nossas emoções e opiniões pertinentes ao mundo que nos rodeia (o contexto de cultura). Assim é que se trata de uma categoria não só subjetiva, mas também socialmente compartilhada.

SILVA $(2019,96)$

\footnotetext{
${ }^{11}$ De acordo com Bauer \& Arts (2000, p. 56), no clássico capítulo intitulado "A construção do corpus: um princípio para a coleta de dados qualitativos", o termo "saturação" caracteriza "um procedimento para se superar o paradoxo do corpus teórico". Sempre segundo os dois autores, "existem ambientes sociais velhos e novos que estão emergindo em uma sociedade dinâmica. Isto exige uma imaginação sociológica e um conhecimento histórico para se reconhecer novos ambiente sociais"(p. 57), o que se coaduna com o novo cenário que nos exige uma nova leitura, frente às mudanças que o "corona vírus" desafia o nosso planeta, a começar pelo sistema de saúde.
} 
Os critérios de inclusão das fontes estudadas foram artigos completos, pertencentes ao mesmo contexto de cultura, os quais se encontram disponíveis eletronicamente nas bases de dados selecionadas, publicados nos idiomas inglês e português, no período compreendido entre 2010-2020, com a temática proposta no título, resumo ou descritores. Foram excluídos artigos em duplicidade e aqueles que não abordavam a temática proposta.

O levantamento dos artigos foi realizado em março de 2020. As palavras-chave foram experiência do paciente ("patient experience"), cuidados em saúde (healthcare) e inteligência artificial ("artificial intelligence"), com o operador booleano AND entre cada palavra. A pesquisa foi realizada por acesso on-line e as amostras examinadas permitiram-me selecionar cinco artigos que transformei em dados após aplicar os critérios de inclusão.

A captação das informações dos artigos, transformados em dados para o estudo ora apresentado, foi realizada, mediante a utilização do banco de dados elaborado no software Microsoft Office Excel 2013, que contemplou as seguintes variáveis:

a) título do artigo;

b) ano de publicação;

c) base de dados;

d) delineamento do estudo e considerações finais.

Os dados foram analisados com base na literatura da área. A apresentação dos resultados e discussão das informações foi feita de maneira descritiva e interpretativa, dentro dos moldes de uma pesquisa de natureza qualitativa.

\section{Resultados}

Na presente revisão de literatura, foram analisados cinco artigos que atenderam aos critérios de inclusão previamente estabelecidos. A seguir apresenta-se um cenário geral dos dados documentais analisados.

Os artigos selecionados neste estudo encontram-se na base de dados MEDLINE (100\%). Apreciemos, a seguir, o QUADRO I: A BASE DE DADOS, que envolve os títulos dos artigos científicos selecionado para o presente estudo. 
QUADRO I: A BASE DE DADOS

\begin{tabular}{|l|l|}
\hline 1 & $\begin{array}{l}\text { Digital Transformation in Healthcare - Architectures of Present and Future } \\
\text { Information Technologies }\end{array}$ \\
\hline 2 & The future of Obstetrics/Gynecology in 2020: a clearer vision \\
\hline 3 & $\begin{array}{l}\text { Use of Sentiment Analysis for Capturing Patient Experience From Free-Text } \\
\text { Comments Posted Online }\end{array}$ \\
\hline 4 & $\begin{array}{l}\text { Understanding safety-critical interactions with a home medical device through } \\
\text { Distributed Cognition }\end{array}$ \\
\hline 5 & Peri-operative Care Pathways: Re-Engineering Care to Achieve the 'Triple Aim $^{19}$ \\
\hline
\end{tabular}

Todos os trabalhos encontrados são dos últimos sete anos, uma vez que não havia publicação anterior a esse período, pelo menos no que concerne ao tema do presente estudo. Em relação ao tipo de periódico científico, nos quais se encontram registrados os artigos incluídos na revisão, três foram publicados em revistas médicas, um em revista biomédica, um em revista de outra área (Química). Quanto ao tipo de delineamento de pesquisa dos artigos selecionados como dados, todos os estudos encontrados na pesquisa são descritivos (100\%). Esse é o tipo de estudo em que o pesquisador não realiza intervenção no assunto analisado, mas já sugere alguma intervenção, o que sinaliza uma possibilidade de transformação com relação ao tema: a experiência do paciente. Isso incentiva o caminho do trabalho ora apresentado, como poderá ser observado a seguir.

No entanto, todos os trabalhos discutiram a relevância do uso de novas tecnologias na experiência do paciente. Um artigo, porém, evidenciou a transformação digital nas empresas como ponto chave para adaptação das instituições de saúde às demandas individuais do paciente e criação de novos modelos de negócio ${ }^{15}$. Dois estudos destacam a importância do triple aim como meta para melhoria da assistência à saúde frente aos desafios dos avanços tecnológicos ${ }^{16,19}$. Um dos estudos apontou a participação crescente das plataformas online como espaço de expressão dos sentimentos do paciente acerca da experiência vivida nos cuidados em saúde e a análise da sua avaliação a partir de ferramenta de inteligência artificial ${ }^{17}$. Outra pesquisa abordou a necessidade de planejamento de tecnologias que considerem seu contexto de uso para melhor experiência do paciente com dispositivos médicos portáteis. $^{18}$ 


\section{QUADRO II. DISTRIBUIÇÃO DOS ARTIGOS INCLUÍDOS NA REVISÃO}

\begin{tabular}{|c|c|c|c|c|}
\hline Título & Ano & $\begin{array}{c}\text { Base de } \\
\text { Dados }\end{array}$ & Delineamento & Conclusão \\
\hline $\begin{array}{l}\text { Digital } \\
\text { Transformation in } \\
\text { Healthcare - } \\
\text { Architectures of } \\
\text { Present and Future } \\
\text { Information } \\
\text { Technologies }\end{array}$ & 2019 & MEDLINE & Descritivo & $\begin{array}{l}\text { Atualmente, os prestadores de cuidado } \\
\text { em saúde encontram o mesmo desafio: } \\
\text { melhorar os desfechos dos pacientes } \\
\text { enquanto reduz gastos. Além disso, } \\
\text { surgem demandas como manejo de } \\
\text { doenças crônicas da população, } \\
\text { inovações tecnológicas e pacientes } \\
\text { empoderados na própria experiência } \\
\text { de saúde. À medida que tecnologias } \\
\text { como big data, inteligência artificial e } \\
\text { machine learning se consolidam pela } \\
\text { prática, as instituições devem se } \\
\text { adaptar para participar da inovação } \\
\text { expoente. A transformação digital é a } \\
\text { chave para instituições se } \\
\text { flexibilizarem à essa mudança de } \\
\text { maneira inteligente, com uso de } \\
\text { plataformas que auxiliem o manejo } \\
\text { dos pacientes, funcionários e processos } \\
\text { de gestão }\end{array}$ \\
\hline $\begin{array}{l}\text { The future of } \\
\text { obstetrics/gynecology } \\
\text { in 2020: a clearer } \\
\text { vision. } \\
\text { Transformational } \\
\text { forces and thriving in } \\
\text { the new system. }\end{array}$ & 2015 & MEDLINE & Descritivo & $\begin{array}{l}\text { O artigo sugere mudanças para atender } \\
\text { as demandas futuras de assistência à } \\
\text { saúde da mulher. Essas mudanças } \\
\text { devem ter como base o Triple AIM e } \\
\text { as transições dos modelos de } \\
\text { pagamento e prestação de cuidado. Os } \\
\text { autores discutem o uso de tecnologias } \\
\text { clínicas como parte de um novo } \\
\text { sistema de saúde, que requer uma } \\
\text { transformação digital para melhorar a } \\
\text { comunicação, a disponibilidade de } \\
\text { informações e as avaliações do } \\
\text { cuidado clínico. Isso permitiria um } \\
\text { tratamento individualizado com custos } \\
\text { reduzidos e informações } \\
\text { compartilhadas entre médicos e }\end{array}$ \\
\hline
\end{tabular}




\begin{tabular}{|c|c|c|c|c|}
\hline & & & & $\begin{array}{l}\text { pacientes. Os autores concluem que, } \\
\text { para se adaptar ao novo sistema, os } \\
\text { profissionais de Ginecologia e } \\
\text { Obstetrícia, além de ter características } \\
\text { como liderança e compaixão, devem } \\
\text { ficar confortáveis com o uso de } \\
\text { tecnologia. }\end{array}$ \\
\hline $\begin{array}{l}\text { Use of Sentiment } \\
\text { Analysis for } \\
\text { Capturing Patient } \\
\text { Experience From } \\
\text { Free-Text Comments } \\
\text { Posted Online }\end{array}$ & 2013 & MEDLINE & Descritivo & $\begin{array}{l}\text { O trabalho demonstra que é possível } \\
\text { analisar os sentimentos dos pacientes } \\
\text { incorporados aos comentários postados } \\
\text { online sobre sua experiência em } \\
\text { diferentes aspectos do cuidado } \\
\text { prestado pelo hospital. O uso do } \\
\text { machine learning para análise e } \\
\text { predição das avaliações estabelece } \\
\text { relações e semelhanças com a } \\
\text { experiência dos pacientes medida por } \\
\text { métodos tradicionais, como } \\
\text { questionários. Além disso, há na } \\
\text { literatura um interesse crescente na } \\
\text { compreensão do ponto de vista dos } \\
\text { pacientes a partir de suas postagens em } \\
\text { mídias sociais, blogs e sites de } \\
\text { avaliação. O estudo apresenta } \\
\text { possibilidades futuras para } \\
\text { compreender o desempenho dos } \\
\text { sistemas de saúde. }\end{array}$ \\
\hline
\end{tabular}




\begin{tabular}{|c|c|c|c|c|}
\hline $\begin{array}{l}\text { Understanding safety- } \\
\text { critical interactions } \\
\text { with a home medical } \\
\text { device through } \\
\text { Distributed Cognition. }\end{array}$ & 2015 & MEDLINE & Descritivo & $\begin{array}{l}\text { Uma vez que os cuidados em saúde } \\
\text { têm migrado do hospital para o lar, se } \\
\text { mostra cada vez mais importante } \\
\text { entender como pacientes interagem } \\
\text { com dispositivos médicos em casa. } \\
\text { Nesse estudo foi aplicada uma } \\
\text { ferramenta teórica (Distributed } \\
\text { Cognition) em entrevistas para } \\
\text { entender interações de pacientes } \\
\text { portadores de doença renal com uma } \\
\text { tecnologia de hemodiálise em casa } \\
\text { (Home Hemodialysis Technology), e } \\
\text { aspectos que poderiam fornecer uma } \\
\text { melhor experiência do paciente ao } \\
\text { utilizar esses equipamentos. Os autores } \\
\text { concluem que para planejar } \\
\text { dispositivos de uso claro e seguro, há a } \\
\text { necessidade de uma tecnologia que } \\
\text { leve em consideração os cenários e } \\
\text { contextos do seu uso. }\end{array}$ \\
\hline $\begin{array}{l}\text { Peri-operative Care } \\
\text { Pathways: Re- } \\
\text { Engineering Care to } \\
\text { Achieve the 'Triple } \\
\text { Aim' }\end{array}$ & 2019 & MEDLINE & Descritivo & $\begin{array}{l}\text { Os profissionais de saúde encaram o } \\
\text { desafio do triple aim, realizado pelo } \\
\text { Institute for Healthcare Improvement, } \\
\text { como oportunidade de melhorar a } \\
\text { experiência do paciente e saúde da } \\
\text { população enquanto reduz custos. A } \\
\text { organização eficaz destas metas será } \\
\text { possível através da coleta de dados, } \\
\text { que deve ser apoiada por ferramentas } \\
\text { para análise. Os autores acreditam que } \\
\text { para a especialidade médica de } \\
\text { anestesia, esse desafio leva a uma } \\
\text { mudança decisiva na experiência do } \\
\text { paciente, contribuindo para } \\
\text { transformar a saúde e o valor do } \\
\text { cuidado prestado com cuidados } \\
\text { padronizados e individualizados e, ao } \\
\text { mesmo tempo, enfrentar os desafios } \\
\text { associados às mudanças tecnológicas e } \\
\text { nas formas de trabalho. }\end{array}$ \\
\hline
\end{tabular}




\section{Discussão}

Os resultados encontrados indiciam uma relativa escassez de produção científica relativa à temática investigada. Não obstante, seus autores tratam o tema como relevante dentro do contexto de transformação digital na qual se insere a saúde mundial, ao considerar o avanço tecnológico como possível responsável pela reestruturação das organizações de saúde de modo a se adaptarem às necessidades do paciente.

Algumas observações tornam-se, aqui, necessárias. A base de dado LILACS não contribuiu com resultados para o estudo. Essa base de dados, por reunir literatura latino-americana, poderia fornecer resultados próximos a realidade do Brasil. Além disso, é um tema de discussão recente, não há publicações anteriores ao ano de 2013. Novamente fica demonstrada a necessidade de estudos sobre o tema.

No que concerne ao objetivo desta revisão, ou seja, examinar o impacto da inteligência artificial na experiência do paciente, parece haver concordância entre os autores sobre o uso de ferramentas tecnológicas para individualizar a jornada do paciente e a assistência à saúde enquanto otimizam gastos e entregam resultados com excelência clínica ${ }^{15,16,17,19}$. As propostas de desenvolvimento da qualidade, consequentemente, podem ser abordadas em múltiplos ângulos, desde experiências de atendimento até medidas e indicadores de gestão do processo clínico'

A análise da jornada do paciente é uma ferramenta de gestão que, de forma prática, abrange esforços para melhoria e inovação em todos os pontos do atendimento ${ }^{1}$. Essas mudanças levam instituições de saúde a empreender nas metas do triple aim com intuito de aprimorar a coordenação do cuidado e direcionar recursos para medidas de melhoria efetiva da saúde ${ }^{16,19}$.

A existência de uma plataforma tecnológica de informação possibilita que todos os processos, custos e resultados dos pacientes sejam assimilados e integrados para oportunizar a mensuração de resultados e custos ao longo da jornada ${ }^{20}$. O uso da Inteligência Artificial em Medicina, através de processamento de dados e algoritmos pré-definidos, demonstra novas soluções para problemas médicos e de gestão, além de possibilitar diagnóstico preciso e tratamento de problemas de saúde individuais e coletivos $^{21}$. 
Atualmente, o uso de tecnologias para acessar informações em saúde é enfatizado pelo aumento no uso de celulares e aplicativos como ferramentas significativas de comunicação entre familiares, amigos e prestadores de serviço ${ }^{11}$. As instituições de saúde que atuam no online são um espaço de geração, agregação e distribuição de conteúdo de saúde em vários canais digitais; isso representa um grande potencial tecnológico para assistência à saúde. A ampla popularidade e uso das plataformas digitais devem torná-las cada vez mais necessárias para promoção da saúde ${ }^{18,23}$.

O desenvolvimento das tecnologias oportuniza o delineamento de sistemas de apoio à decisão clínica durante o cuidado à saúde, prontuário acessível para os usuários e a extração fácil de dados. Com o tempo, o processo de mensuração de resultados e a experiência no cuidado da condição de saúde produz um estímulo ao aprimoramento e à inovação, criando um ciclo efetivo de valor ${ }^{21}$. Assim, fica demonstrado que o uso da tecnologia pode promover segurança e bem estar do paciente, além de melhorar a qualidade do atendimento.

\section{Considerações finais}

As publicações acerca do tema ainda se mostram incipientes; porém, em termos de valorização da experiência do paciente, pode-se considera-las relevantes do ponto de vista social e econômico por transformação intensa e crescente no atual contexto de saúde e no que diz respeito à assistência à melhoria da saúde. Espera-se que o estudo sirva como base para que novas pesquisas sejam realizadas, sobretudo com dados empíricos, que permitam mensurar a experiência do paciente, para se chegar a outra visão da questão.

\section{Referências}

1. WOLF, J. A. PhD; Niederhauser, V. DrPH, RN; Marshburn, Dianne PhD, RN, NE-BC; and LaVela, Sherri L. PhD, MPH, MBA "Defining Patient Experience," Patient Experience Journal: Vol. 1: Iss. 1, Article 3. Available at:http://pxjournal.org/journal/vol1/iss1/3. 2014.

2. THE BERYL INSTITUTE website, Defining Patient Experience. Dísponível em: http://www.theberylinstitute.org/?page=DefiningPatientExp. Acesso em Janeiro de 2020. 
3. INSTITUTE FOR HEALTHCARE IMPROVEMENT website. Disponível em: www.IHI.org. Acesso em Janeiro de 2020

4. BERWICK, D. M., Nolan, T. W., \& Whittington, J. The triple aim: Care, health, and cost. Health Affairs, 27(3), 759-769. https://doi.org/10.1377/hlthaff.27.3.759. 2008

5. STIEFEL M, Nolan K. A Guide to Measuring the Triple Aim: Population Health, Experience of Care, and Per Capita Cost. IHI Innovation Series white paper. Cambridge, Massachusetts: Institute for Healthcare Improvement; 2012.

6. LUCIAN LEAPE INSTITUTE. Through the eyes of the workforce: creating joy, meaning and safer health care. Boston, MA: National Patient Safety Foundation. 2013

7. VARKEY P, Reller MK, Resar RK. Basics of quality improvement in health care. Mayo Clin Proc.;82(6):735-739. doi:10.4065/82.6.735. 2007

8. NEVILLE, R. Transformação digital para saúde: automação de processos e gestão digital para hospitais. Pixeon. 2019. Dísponivel em: https://www.pixeon.com/blog/transformacao-digital-para-saude/. Acesso em: fevereiro de 2020

9. LUXFORD, K., \& Sutton, S. How does patient experience fit into the overall healthcare picture. Patient Experience Journal, 1(1), 20-27. https://doi.org/10.35680/2372-0247.1002. 2014

10. KAPLAN ANDREAS. Siri, Siri, in my hand: Who's the fairest in the land? On the interpretations, illustrations, and implications of artificial intelligence. Business Horizons 62, 15-25. 2019

11. MILANI R. V., Franklin Nina C., The Role of Technology in Healthy Living Medicine, Progress in Cardiovascular Diseases. doi: 10.1016/j.pcad.2017.02.001; 2017.

12. CALDEIRA, H. A inteligência artificial aplicada na medicina. CM tecnologia website. Disponível em: https://cmtecnologia.com.br/blog/inteligenciaartificial/; 2017

13. BAUER, M.; BAUER, A. A construção do corpus: um princípio para a coleta de dados qualitativos. In: BAUER, M. W.; GASKELL, G. Pesquisa qualitativa com texto, imagem e som: um manual prático. Tradução Pedrinho A. Guareschi. 10. ed. Petrópolis, RJ: Vozes, 2002. 
14. ULBRA. Sistema de Bibliotecas da Universidade Luterana do Brasil. Disponível em: https://www.ulbra.br/ead/bibliotecas. Acesso em Mar de 2020

15. SILVA, D.E.G. Contexto social entre caminhos teórico-metodológicos transdisciplinares, p. 95-111. In: D.E.G. da Silva (org.), Análise de Discurso, Gramática e Contexto Social. Brasília, DF: Thesaurus Editora, 2019

16. GOPAL G, Suter-Crazzolara C, Toldo L, Eberhardt W. Digital transformation in healthcare - architectures of present and future information technologies. Clin Chem Lab Med.; 57(3):328-335. doi:10.1515/cclm-20180658; 2019

17. LAGREW DC Jr, Jenkins TR. The future of obstetrics/gynecology in 2020: a clearer vision. Transformational forces and thriving in the new system. Am J Obstet Gynecol.;212(1):28-33.e1. doi:10.1016/j.ajog.2014.08.022; 2015.

18. GREAVES F, Ramirez-Cano D, Millett C, Darzi A, Donaldson L. Use of sentiment analysis for capturing patient experience from free-text comments posted online. J Med Internet Res.;15(11):e239. doi:10.2196/jmir.2721; 2013.

19. RAJKOMAR A, Mayer A, Blandford A. Understanding safety-critical interactions with a home medical device through Distributed Cognition. $J$ Biomed Inform.; 56:179-194. doi:10.1016/j.jbi.2015.06.002; 2015

20. GROCOTT MPW, Edwards M, Mythen MG, Aronson S. Peri-operative care pathways: re-engineering care to achieve the 'triple aim'. Anaesthesia; 74 Suppl 1:90-99. doi:10.1111/anae.14513; 2019.

21. PORTER, M. E.; LEE, T. H. The Strategy That Will Fix Healthcare. Harvard Business Review, v. 1277, n. October, p. 1-19; 2013.

22. SZOLOVITS P. Artificial intelligence and medicine. Artificial Intelligence in Medicine, 41(2), 1-19. https://doi.org/10.4324/9780429052071-1;2019. 